\title{
Endoscopic Facial Rejuvenation: Endoforehead the Functional Lift-Brief Update
}

\author{
Nicanor Isse ${ }^{1}$
}

Published online: 5 August 2020

(C) Springer Science+Business Media, LLC, part of Springer Nature and International Society of Aesthetic Plastic Surgery 2020

This journal requires that authors assign a level of evidence to each article. For a full description of these EvidenceBased Medicine ratings, please refer to the Table of Contents or the online Instructions to Authors www.springer. com/00266.

\section{Originality}

Even though the technique was "original," at the time of its publication, the basic concept of the procedures was based on very well-established principles of tissue preservation, preservation of circulation (pedicle flap), and decreased tissue traction while obtaining brows and tissue repositioning as a goal.

A minimal incision approach was very well received due to reduced post-surgical scar formation, elimination of the tissue necrosis, remarkable decreased thinning of the hair, diffused alopecia, and reduced postoperative anesthesia (numbness) of the scalp.

The use of an endoscope was essential to visualize blood vessels, sensory and motor nerves branches, muscles, and fatty tissue compartments.

To achieve the brows elevation and reposition, it was necessary to shift the concept of tissue traction to tissue modifications (myotomies and neurotomies) and tissue fixations.

Originally, screws were used as fixation device, and then, it evolved to linear and concentric suture suspension techniques.

\section{Nicanor Isse}

NicIsseMd@icloud.com

1 American Board of Plastic Surgeon, 1441 Avocado Ave. Suite 307, Newport Beach, CA 92660, USA
As depicted in one of the drawing in the original article, a midface approach was also carried out at same time in indicated cases. The endoscopic peri-orbital rejuvenation, endoscopic midface, and endoscopic lower face/neck were presented in several scientific meetings as well as in comprehensive Endoscopic Plastic Surgery Seminars (EPSES) which was given monthly for many years nationally and internationally. The seminars were carried out in two and half days, consisting of comprehensive lectures, live surgical demonstrations, and cadaver dissections.

New instruments were developed to carry out the technique.

Endoscopic tissue elevators and visualization, dissectors, and sutures suspension self-anchoring devices were used to perform minimal endoscopic surgeries.

The concepts of minimal incisional facial rejuvenation, tissue modifications, suture suspensions, and the consequent volumetric changes on the facial tissue have opened a myriad of alternative techniques. They came to enrich the armamentarium of facial rejuvenation and remodeling. Some of the alternative techniques developed at the time of Endoscopic Forehead Lift or later on were: non-endoscopic forehead lift (percutaneous digital-guided dissection); chemical myotomies, energy-based neurotomies, volumetric facial modifications (soft tissue augmentation), thread Lift.

The longevity and minimal morbidity of the endoscopic forehead lift, compare with similar techniques, addressing brow's reposition; the pleasant and harmonious results, as well as, acting as temporal approach to the mid- and lower face, lead this technique to endure over time.

Finally, I would like to acknowledge many of our colleagues (plastic surgeons): the late Williams Shaw, M.D.; 
Peter Fodor, M.D.; Gordon Sasaki, M.D.; James Watson, M.D.; Chia Chi Kao, M.D.; ND Moscoe, M.D.; and Alessandro Gennai, M.D., for their participations, collaborations, and criticisms during the development of the technique allowing the original concept and procedure to evolve into a safer and predictable outcome.

Publisher's Note Springer Nature remains neutral with regard to jurisdictional claims in published maps and institutional affiliations. 